\title{
Attenuation and Shielding Performance of Wood-Polymer Composites Synthesized via Melt-Blend Technique
}

\author{
Abubakar Yakubu1 ${ }^{*}$, Zulkifli Abbas², Abdullahi Sirajo' ${ }^{1}$ \\ ${ }^{1}$ Department of Physics, Faculty of Science, Kebbi State University of Science and Technology, Aliero, Nigeria \\ ${ }^{2}$ Department of Physics, Faculty of Science, Universiti Putra Malaysia, Serdang, Selangor Darul Ehsan, Malaysia \\ Emai:*abulect73@yahoo.com
}

How to cite this paper: Yakubu, A., Abbas, Z. and Sirajo, A. (2020) Attenuation and Shielding Performance of Wood-Polymer Composites Synthesized via Melt-Blend Technique. Open Access Library Journal, 7: e5444.

https://doi.org/10.4236/oalib.1105444

Received: May 6, 2019

Accepted: February 14, 2020

Published: February 17, 2020

Copyright $\odot 2020$ by author(s) and Open Access Library Inc.

This work is licensed under the Creative Commons Attribution International License (CC BY 4.0).

http://creativecommons.org/licenses/by/4.0/

\begin{abstract}
In the recent, filler-polymer composites researches have been on the rise due to its implication to electromagnetic interference (EMI) applications. The composition, morphology and surface rheology of the filler-polymer composites play an immense role in determining the electrical, mechanical, and optical properties. In this paper, the preparation and characterization of micro-composites of wood (biodegradable waste material)/polycaprolactone (PCL) composites are reported. The micro-composites were prepared using melt blend technique via the solid state route. This method was selected because of its numerous advantages compared to other methods such as being easy, cheap with mass production of materials. To determine attenuation, rectangular waveguide (RWG) method was used. The magnitudes of the transmission coefficient $\left(S_{21}\right)$ from the waveguide measurement were used to determine the attenuation of the wood-PCL composite substrate with respect to different percentages of wood filler. Result from the measurement showed amongst others that as the filler content increases, the attenuation increases. The highest magnitude for attenuation was calculated for the $62.5 \%$ wood-PCL composites with a value of $-6.46 \mathrm{~dB}$ and the least attenuation was calculated for the $12.5 \%$ wood micro filler, which value gave $-3.34 \mathrm{~dB}$ which is good for low shielding applications. Scanning electron microscope (SEM) was used to study the surface morphology of the samples.
\end{abstract}

\section{Subject Areas}

Composite Material, Computational Physics, Experimental Physics, Material Experiment

\section{Keywords}

Composites, Wood Filler, Attenuation, Shielding, Microwave 


\section{Introduction}

Most applications in engineering do deal with minimum shielding effectiveness. For this singular reason, the understanding and selection of materials for effective shielding are vital in both design and fabrication. Material behaviour to electromagnetic interference field is of immense importance, especially in areas of medical applications, military hardware, electronics, telecommunication industries and other shielding applications. Most casing or packaging for electrical and electronic devices is polymeric in nature. High amongst polymer characteristics are simplicity of fabrication, light weight, low cost, and excellent insulating property and these have made plastics one of the most suitable materials for electrical and electronics applications. The function of plastics in electrical and electronic applications was limited to no-load bearing general-purpose applications [1].

Based on this quality of polymer, the scientific community has delved into numerous researches to understanding polymeric compounds and substrates. With this development, polymer composite is the major player in the fabrication of novel materials good for a variety of different applications, such as electrical engineering and high frequency devices like microwave especially to shielding applications, microwave absorption and electromagnetic interference (EMI), electrodes and sensors [2].

The reinforcement of polymers using different types of organic or inorganic fillers is common practice in the production of systems with improved mechanical, dielectric properties [3]. Micrometer-sized inorganic particles are commonly used for the reinforcement of polymer matrices to improve thermal conductivity, and meet mechanical requirements. Properties of the polymer composites are affected by factors, like inherent characteristics of each component, contact, dimension and shape of the fillers, and the nature of their interfaces [4].

Knowledge of materials behavior placed in an electromagnetic field is of immense importance especially when it relates to military hardware, electronics, communication, and industrial applications and shielding [5]. The measurement of transmission coefficient of materials in the microwave frequency range is found in several areas. It is vital that transmission coefficient measurement is fully understood so as to enable satisfactory attenuation measurements of materials good for microwave absorption. Attenuation is a function which can be affected by a group of factors. For this study, concentration is based on the effect of the filler content on the matrix.

In this work, wood-polymer composite structures were synthesized, and investigation on electromagnetic propagation, absorption properties of the substrate at $8-12 \mathrm{GHz}$ with thickness of $10 \mathrm{~mm}$ was carried out. The microwave properties of samples were investigated by using rectangular waveguide connected with vector network analyzer via coaxial cable. Attenuation of wood-PCL micro-composites at filler contents of $(12.5 \%, 22.5 \%, 37.5 \%, 50 \%$, and $62.5 \%)$ were investigated in this work. The total volume fraction of the composites was set at $40 \mathrm{~g}$. 


\section{Sample Preparation}

\section{Wood-Polymer Composites}

Wood polymer composites materials were prepared via melt-blend technique by mixing the wood filler to PCL. The materials were weighed according required composition (Table 1). For the preparation of the samples used in this study, the followings were used; wood dust (99.9\%) purity, (Sigma, Aldrich), Polycaprolactone, PCL $\left(\mathrm{C}_{6} \mathrm{H}_{10} \mathrm{O}_{2}\right) 97.0 \%$ purity, with density of $1.146 \mathrm{~g} / \mathrm{cm}^{3}$ (Sigma Aldrich).

The wood-PCL composites was prepared via the melt blend technique using the Thermo Haake extruder Polydrive three-phase motor with a drive of $1.5 \mathrm{~kW}$, $3 \times 230 \mathrm{~V}, 40 \mathrm{~A}$ and speed range of $0-120 \mathrm{rpm}$. In this method, the Thermo Haake machine is set to desire heating temperature and allowed to heat up. All other components of the machine are set following recommended settings. For our method, the machine is set to $90^{\circ} \mathrm{C}$ for heating, the rotation of the rotors was set to $50 \mathrm{rpm}$. After the machine has reached the required temperature $\left(80^{\circ} \mathrm{C}\right)$ because it was close to the melt point $(60-70)^{\circ} \mathrm{C}$ of PCL.

The polycaprolactone (PCL) is poured into the vial of the Thermo Haake heating block. After 5 minutes, the wood dust is introduced into the vial. The mixture is left for another 20 minutes before taken out and fabricated into desired dimension using hot and cold press. Table 1 illustrates the ratio of blending.

\section{Experimental}

The material under test is inserted in a piece of transmission line, and the properties of the material are deduced on the basis of the rejection from the material and the transmission through the material [6] [7]. The fringes of the field of reflection due to this material are used to calculate its transmission and reflection coefficients. The method of using transmission lines in measurement is for accuracy of results since microwave guides have field focusing ability [8]. For this study, sample is placed between two waveguides and excited with electromagnetic radiation.

For the transmission coefficient measurement, a $10 \mathrm{~mm}$ sample was placed carefully inside the RWG which then connected via a coaxial cable to network

Table 1. Composition of samples prepared.

\begin{tabular}{rc}
\hline Filler \% & PCL \% \\
$12.5(5 \mathrm{~g})$ & 87.5 \\
$22.5(10 \mathrm{~g})$ & 77.5 \\
$37.5(15 \mathrm{~g})$ & 62.5 \\
$50.0(20 \mathrm{~g})$ & 50.0 \\
$62.5(25 \mathrm{~g})$ & 37.5 \\
\hline
\end{tabular}


analyzer (Agilent 8570B). For instrument validity, the waveguide was used to measure air and PTFE since both are standard materials. The specimen dimensions are machined to fit as tightly as possible into the waveguide removing any possibility for air gap between the sample and the guide around the sample walls.

Shown in Figure 1 is the measurement set up, showing the RWG.

Figure 2 is the measured scattering parameter for the standard material (air). The result showed that the transmission coefficient is higher in magnitude than the reflection coefficient. Theoretically, the value of the transmission coefficient should be close to unity [9].

The scattering or $S$ parameters of a two-port network are defined in terms of the wave variables [10].

Of importance is the nomenclature used for the scattering parameters. The parameters $S_{11}$ and $S_{22}$ are called the reflection coefficients, whereas $S_{12}$ and $S_{21}$ are called the transmission coefficients. These are the parameters directly measurable at microwave frequencies. Generally, the $S$ parameters are written in complex form as [10];

$$
S_{g g}=\frac{b_{g}}{a_{g}}(g=1,2)
$$

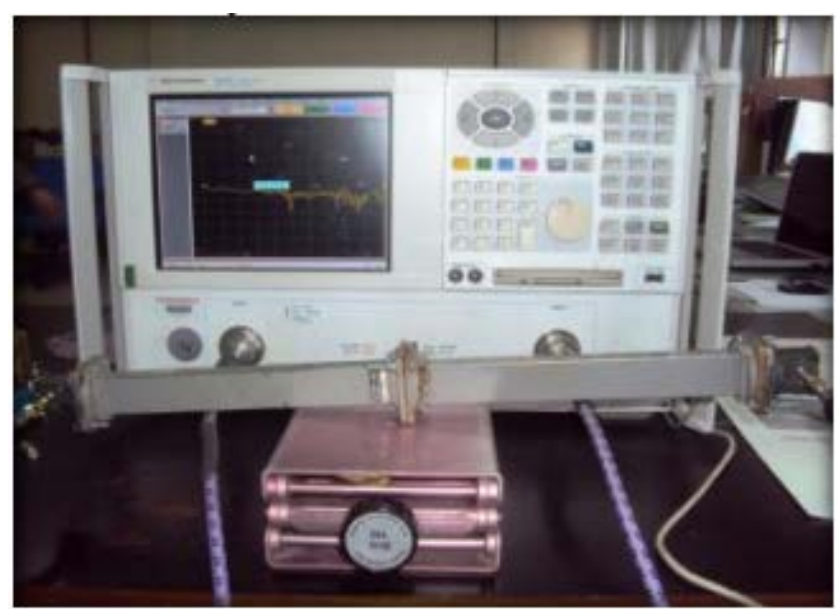

Figure 1. Measurement set up for $S$-parameters.

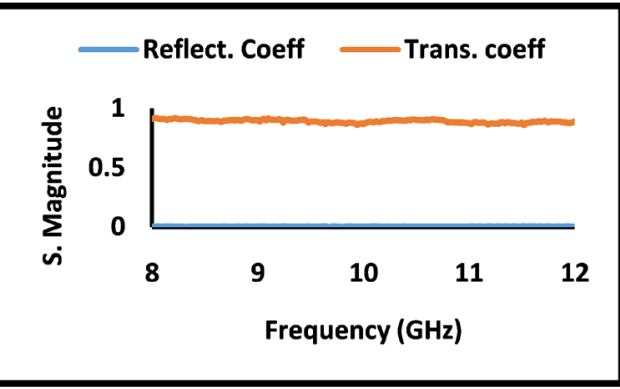

Figure 2. Measured $S$-parameter for standard material (air). 


$$
S_{p g}=\frac{b_{p}}{a_{p}} \quad(p \neq g ; p=1,2 ; g=1,2)
$$

The reflection coefficient;

$$
\Gamma_{g}=S_{g g}=\frac{b_{g}}{a_{g}}
$$

The transmission coefficient;

$$
T_{g \rightarrow p}=S_{P P}=\frac{b_{p}}{a_{p}}
$$

The result obtained via the Vector Network Analyzer (VNA) for the transmission coefficients $\left(S_{21}\right)$ is then used to compute the attenuation of the material under study using Equation (4).

$$
\text { Attenuation }(\mathrm{dB})=-20 \log \left(S_{21}\right)
$$

\section{Experimental Results}

After carrying out the measurements, the raw data were used to plot a graph of $S_{21}$ against frequency.

Careful observation on Figure 3, shows that the transmission coefficients with respect to wood filler content sequential decreases as content increases. Thus, transmission coefficient decreases with increased wood content. This behavior is in agreement with theory that states that denser materials decrease transmission [11]. The highest value of transmission coefficients is recorded for the lowest ratio of filler at $5 \mathrm{~g}$ wood filler with the least value recorded for the highest ratio of filler at $25 \mathrm{~g}$ content. This result is in agreement with impedance mismatch theory, where materials with the highest permittivity depict lower transmission coefficient values [12].

Further observation in Figure 3 showed that transmission coefficient decreases as frequency increases for all samples. This might be attributed to perfect dispersion of the filler in the matrix. So the result, confirmed homogeneity of the particles dispersion in the matrix which will tend to increase or decrease the transmission of the electromagnetic radiation depending on the dispersion of wood dust in the matrix. However, the general result shows that the increase in

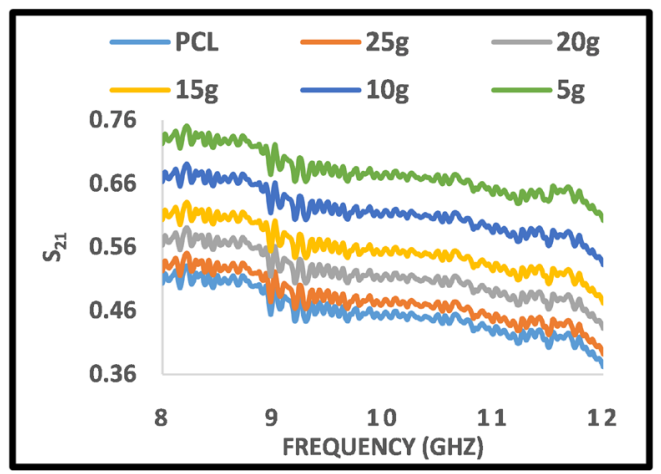

Figure 3. Measured $S_{21}$ for all samples. 
filler content decreases the magnitude of the transmission coefficient for all filler composition at all frequency ranges as evident in Figure 4.

Furthermore, the sinusoidal-like wave form over all frequency ranges was attributed to the impedance mismatched between the input impedance of the waveguide and the surface impedance of the sample as well as the characteristic impedance of the coaxial cable [13] [14].

To calculate the attenuation of the different wood-polymer pellets, raw data of the transmission coefficients was used. Equation (4) was then used to calculate the attenuation. The calculated result is then interpreted in Figure 5. Observation on Figure 5 shows that the least attenuation was calculated for the $12.5 \%$ wood filler content whereas the highest attenuation was calculated for the $62.5 \%$ filler content.

This result is in agreement with $S_{21}$ since the least transmitted composites have the largest absorption.

Figure 6 depicts the mean value of attenuation for all filler percentage. The graph shows a continued increase in attenuation as filler content increases. This behavior is in agreement with theory that states that, denser materials decrease transmission [15]. This behaviour might be attributed to the perfect dispersion

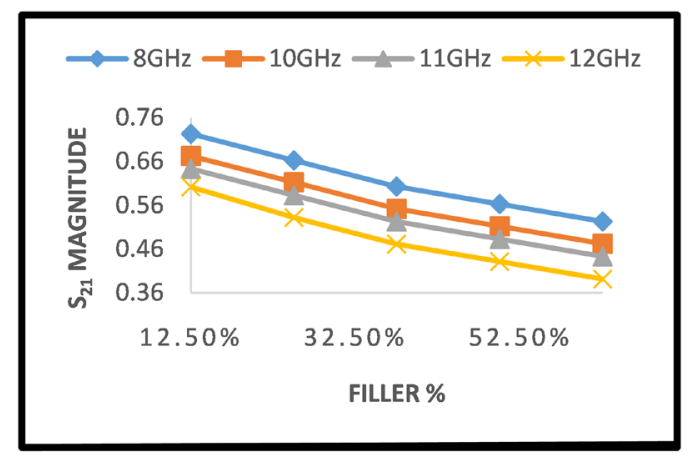

Figure 4. Wood filler content vs transmission coefficients.

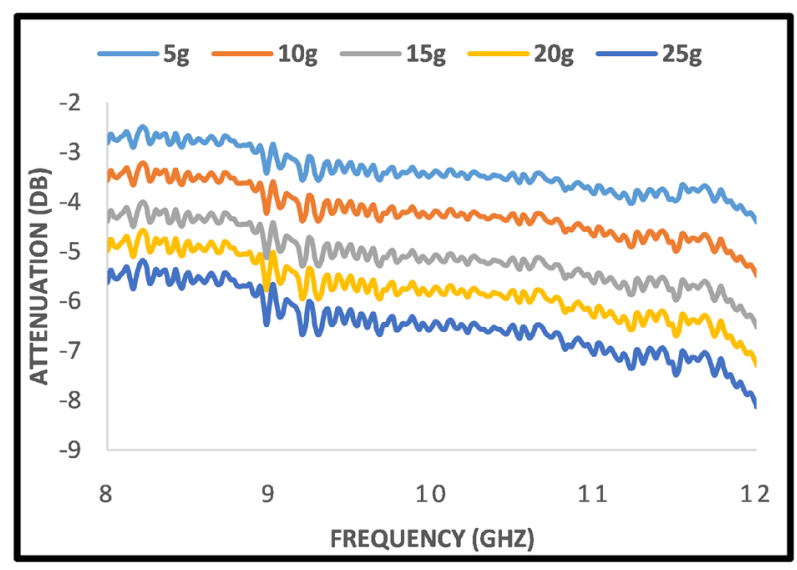

Figure 5. Calculated attenuation of different composition of wood-PCL composites. 
of the filler in the matrix, as the homogeneity of the particle dispersion in the matrix will tend to increase or decrease the attenuation of the EM radiation depending on the dispersion of the wood particles in the matrix [16].

\section{SEM Micrographs}

The filler composites were analyzed using scanning electron microscopy (SEM) with a field emission gun and an accelerating voltage of $10 \mathrm{kV}$. Shown in Figure 7 is the micrographs for the $50 \%$ filler content. Complete dispersion of the wood dust into the polymer can be observed and this is attributed to good sample preparation technique.

Further observation reveals that wood particles are indeed dispersed as discrete particles in the polymer matrix. The compact wood particles are fully integrated in the composites on the entire fractured surface. The morphology indicates that wood particles have indeed reacted with the PCL evenly to produce wood-PCL composites [17].

\section{Conclusions}

The synthesis of wood-polymer was successfully achieved in this work. Investigation on the propagation of electromagnetic radiation through an RWG and

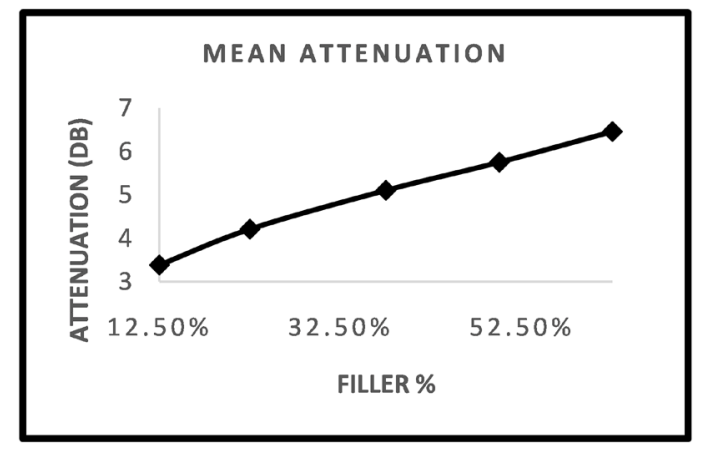

Figure 6. Mean attenuation of the Wood-PCL composites.

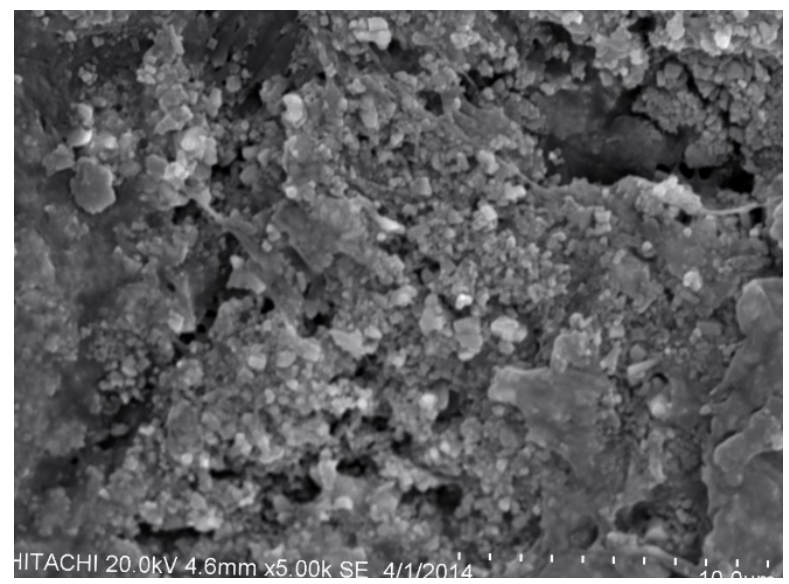

Figure 7. SEM micrographs of 50\% filler composites. 
determination of material attenuation were carried out at microwave frequency. The work was limited to transmission coefficient, attenuation and surface morphology of the samples prepared.

Since shielding effectiveness is the process of using specialized materials to reduce EMI field or waves that enter a specific enclosure.

It is concluded that the material produced is good for microwave shielding applications in the range of 3.4 to $6.5 \mathrm{~dB}$.

Based on the results obtained, the materials produced in this work can be tailored for electronics, telecommunication and medical industries.

\section{Conflicts of Interest}

The authors declare no conflicts of interest regarding the publication of this paper.

\section{References}

[1] Roffi, M., Ferreira, F.J. and De Almeida, A.T. (2017) Comparison of Different Cooling Fan Designs for Electric Motors. IEEE International Electric Machines and Drives Conference, Miami, 21-24 May 2017, 1-7. https://doi.org/10.1109/IEMDC.2017.8002270

[2] Yakubu, A., Abbas, Z. and Mohammad, D.M. (2018) Electromagnetic Characterization and Field Distribution of $\mathrm{Co}_{0.5} \mathrm{Zn}_{0.5} \mathrm{Fe}_{2} \mathrm{O}_{4}$ Using Patch Microstrip Antenna and Finite Element Methods. Equity Journal of Science and Technology, 5, 123-131.

[3] Mittal, G., Rhee, K.Y., Mišković-Stanković, V. and Hui, D. (2018) Reinforcements in Multi-Scale Polymer Composites: Processing, Properties, and Applications. Composites Part B: Engineering, 138, 122-139. https://doi.org/10.1016/j.compositesb.2017.11.028

[4] Haque, M.M., Shamsuzzaman, M., Uddin, M.B., Salahuddin, A.Z.M. and Khan, R.A. (2019) Fabrication and Characterization of Shielding Properties of Heavy Mineral Reinforced Polymer Composite Materials for Radiation Protection. European Journal of Engineering Research and Science, 4, 15-20. https://doi.org/10.24018/ejers.2019.4.3.1132

[5] Joshi, M. and Chatterjee, U. (2016) Polymer Nanocomposite: An Advanced Material for Aerospace Applications. In: Advanced Composite Materials for Aerospace Engineering, Woodhead Publishing, Boston, 241-264. https://doi.org/10.1016/B978-0-08-100037-3.00008-0

[6] Ahmad, A.F., Abbas, Z., Obaiys, S.J. and Abdalhadi, D.M. (2016) Attenuation Performance of Polymer Composites Incorporating NZF Filler for Electromagnetic Interference Shielding at Microwave Frequencies.

[7] Yang, W., Meyers, M.A. and Ritchie, R.O. (2019) Structural Architectures with Toughening Mechanisms in Nature: A Review of the Materials Science of Type-I Collagenous Materials. Progress in Materials Science, 103, 425-483. https://doi.org/10.1016/j.pmatsci.2019.01.002

[8] Yakubu, A., Abbas, Z., Esa, F. and Tohidi, P. (2015) The Effect of ZnO Nanoparticle Filler on the Attenuation of $\mathrm{ZnO} / \mathrm{PCL}$ Nanocomposites Using Microstrip Line at Microwave Frequency. International Polymer Processing, 30, 227-232. https://doi.org/10.3139/217.2993

[9] Pozar, D.M. (2009) Microwave Engineering. John Wiley and Sons, Hoboken. 
[10] Yakubu, A., Abbas, Z. and Hashim, M. (2014) Effect of Material Thickness on Attenuation $(\mathrm{dB})$ of PTFE Using Finite Element Method at X-Band Frequency. Advances in Materials Science and Engineering, 2014, Article ID: 965912. https://doi.org/10.1155/2014/965912

[11] Pozar, D.M. (2016) Microwave Engineering, 2012. John Wiley \& Sons, Hoboken.

[12] Deng, R., Li, M., Muneer, B., Zhu, Q., Shi, Z., Song, L. and Zhang, T. (2018) Theoretical Analysis and Design of Ultrathin Broadband Optically Transparent Microwave Metamaterial Absorbers. Materials, 11, 107. https://doi.org/10.3390/ma11010107

[13] Abbas, Z., Pollard, R.D. and Kelsall, R.W. (2001) Complex Permittivity Measurements at Ka-Band Using Rectangular Dielectric Waveguide. IEEE Transactions on Instrumentation and Measurement, 50, 1334-1342. https://doi.org/10.1109/19.963207

[14] Ahmad, A.F., Abbas, Z., Obaiys, S.J., Ibrahim, N., Hashim, M. and Khaleel, H. (2015) Theoretical and Numerical Approaches for Determining the Reflection and Transmission Coefficients of OPEFB-PCL Composites at X-Band Frequencies. PLoS ONE, 10, e0140505. https://doi.org/10.1371/journal.pone.0140505

[15] An, Y., Sun, Y., Zhang, M., Jiao, J., Wu, D., Wang, T. and Wang, K. (2018) Tuning the Electronic Structures and Transport Properties of Zigzag Blue Phosphorene Nanoribbons. IEEE Transactions on Electron Devices, 65, 4646-4651.

[16] Yakubu, A., Zulkifli, A. and Suleiman, S. (2019) Enhancing the Permittivity, Permeability and Morphology of ZnO-PCL Nanocomposites via Nano Inclusion Treatment. Global Journal of Polymer Science, 1, 1-7.

[17] Yakubu, A., Zulkifly, A. and Sahabi, S. (2019) Structural and Dielectric Evolution of Cobalt Ferrite Nanoparticles for Microwave Applications. Global Journal of Material Science and Engineering, 1, 1-4. 